\section{CPS-138 AN OBSERVATIONAL RETROSPECTIVE STUDY ON TREATMENT ADHERENCE OF LONG ACTING INJECTABLE ANTIPSYCHOTICS}

${ }^{1}$ AM Fernández Varela*, ${ }^{2}$ LM Martínez Agulleiro, ${ }^{1} B$ Salazar Laya, ${ }^{2} D$ Núñez Arias, ${ }^{1}$ I Rodríguez Penín. ${ }^{1}$ Hospital Arquitecto Marcide, Farmacia Hospitalaria, Ferrol-A Coruña, Spain; ${ }^{2}$ Hospital Arquitecto Marcide, Psiquiatría, Ferrol-A Coruña, Spain

\subsection{6/ejhpharm-2020-eahpconf.239}

Background and importance Treatment with long acting injectable (LAI) antipsychotics has been shown to improve treatment adherence compared with oral antipsychotics, but it is still controversial if adherence is modified with the use of polytherapy with oral and LAI antipsychotics.

Aim and objectives To evaluate treatment adherence (monotherapy with LAI antipsychotics versus polytherapy with LAI and oral antipsychotics) in patients with different psychiatric disorders.

Material and methods An observational retrospective study was developed, and two cohorts of patients were defined regarding their antipsychotic therapy: (1) monotherapy with LAI antipsychotics and (2) polytherapy with oral and LAI antipsychotics. Patients who began treatment with LAI antipsychotics before 2016 were included in this study. Adherence to treatment was examined during the year 2018, based on the electronic registration of LAI antipsychotic administrations and oral antipsychotic withdrawals at the pharmacy offices. Optimal adherence was defined as 100\% of prescriptions withdrawals or $100 \%$ of the doses of LAI antipsychotics administered.

Results A total of 73 patients were included (39 with monotherapy, 34 with polytherapy), with a mean age of 57.1 years. The most prevalent diagnosis was schizophrenia (49.3\%), followed by delusional disorder $(17.8 \%)$ and personality disorder (11.0\%). No significant differences were found for sociodemographic data between the groups. Adherence to LAI antipsychotics was $97.3 \%$ in the monotherapy group and $87.1 \%$ in the polytherapy group, with no significant difference between the two groups $(p=0.187)$. Adherence to oral antipsychotics was $63.7 \%$.

Conclusion and relevance Adherence to treatment was suboptimal in both groups, but lower in patients receiving polytherapy with oral and LAI antipsychotics. Treatment adherence decreased as treatment complexity increased, as seen in previous literature.

\section{REFERENCES AND/OR ACKNOWLEDGEMENTS}

No conflict of interest.

\section{CPS-139 PSYCHOTROPIC DRUG USAGE IN OCTOGENARIAN AND NONAGENARIAN COMPLEX CHRONIC PATIENTS}

${ }^{1} \mathrm{~A}$ Perez Contel ${ }^{*},{ }^{1} \mathrm{~S}$ Ortonobes Roig, ${ }^{1} \mathrm{~N}$ Soler Blanco, ${ }^{2} \mathrm{G}$ Diestre Ortin, ${ }^{1} \mathrm{P}$ Miralles-Albors, ${ }^{1} S$ Fernández-Molina, ${ }^{1} \mathrm{~A}$ Barragán Muñoz, ${ }^{1} \mathrm{M}$ Gómez-Valent. ${ }^{1}$ Consorci Corporacio Sanitaria Parc Tauli, Pharmacy, Sabadell, Spain; ${ }^{2}$ Consorci Corporacio Sanitaria Parc Tauli, Psychogeriatrics, Sabadell, Spain

\subsection{6/ejhpharm-2020-eahpconf.240}

Background and importance Elderly patients often manifest behavioural disorders. They commonly involve the use of psychotropic drugs that are associated with drowsiness, confusion and risk of falls, especially in this vulnerable population. The significant increase in psychotropic drug consumption in recent years has promoted strategies to identify potentially inappropriate prescriptions and their optimisation or de-prescription.

Aim and objectives

- To estimate the prevalence of psychotropic drugs at discharge in geriatric patients and describe the most frequently prescribed.

- To evaluate differences between octogenarian and nonagenarian complex chronic patients (CCP).

Material and methods A retrospective observational study was conducted in geriatric patients discharged between May and June 2019 from an acute geriatric unit (41 beds) of a geriatric healthcare centre from a university hospital. Variables registered were age, sex, length of stay (LOS), and number and type of psychotropic drugs at discharge (hypnotics, antidepressants, neuroleptics, mood stabilisers). For octogenarian and nonagenarian CCP designated as primary care, we also collected data on polypharmacy and the Pfeiffer test before admission. Those who died were excluded. Quantitative data are presented as median (Q1-Q3) and we used the MannWhitney-Wilcoxon U test. Statistical analysis was performed with Stata13.

Results A total of 148 patients were included, 87 (58.8\%) women, aged $86(82.75-90.25)$ years. LOS was 9 (6-13.25) days. Prescription of psychotropic drugs was as follows: 68 (49.9\%) patients received hypnotics (49/68 (72.0\%) trazodone and 23/68 (33.8\%) short/intermediate acting benzodiazepines); $46(31.1 \%)$ received antidepressants (15/46 (32.6\%) sertraline); $39(26.3 \%)$ received neuroleptics (25/39 (64.1\%) quetiapine and $13 / 39(33.3 \%)$ risperidone); and 18 (12.2\%) received mood stabilisers (8/18 (44.4\%) gabapentin). The number of patients with at least one psychotropic prescription was 97 $(65.5 \%)$ and $23(15.5 \%)$ had $\geq 3$ psychotropic prescriptions. Differences between octogenarian $(n=29)$ and nonagenarian CCP $(n=20)$ were: LOS $10(7-13)$ versus 8 (5.5-16) days $(\mathrm{p}=0.554)$; number of psychotropic drugs $2(1-3)$ versus 1.5 (0-2) $\quad(\mathrm{p}=0.378)$; polypharmacy $12(10-14)$ versus $11(8.5-$ 12) drugs $(p=0.135)$; and Pfeiffer test $2(0-4)$ versus $3(1-7)$ points $(\mathrm{p}=0.08)$.

Conclusion and relevance

- Two-thirds of patients were receiving treatment with at least one psychotropic drug, with hypnotics and antidepressants the most prescribed.

- The small sample size made it difficult to demonstrate statistically significant differences, but this study suggests that nonagenarian CCP present less polypharmacy and a lower number of psychotropic drugs compared with octogenarian $\mathrm{CCP}$, despite having higher cognitive impairment.

\section{REFERENCES AND/OR ACKNOWLEDGEMENTS}

No conflict of interest.

\section{CPS-140 CURRENT STATUS OF CLINICAL TRIALS FOR ALZHEIMER'S DISEASE}

A Rodriguez*, ME Navarrete-Rouco, P Acin, M Ponce, M Jorques, 0 Ferrandez-Quirante, M Espona. Hospital Del Mar, Pharmacy, Barcelona, Spain

\subsection{6/ejhpharm-2020-eahpconf.241}

Background and importance Alzheimer's disease (AD) is a progressive neurodegenerative process caused by an accumulation of the $A \beta$ amyloid peptide. 
Aim and objectives The objective of this study was to describe the current status of clinical trials (CT) for AD in our hospital pharmacy service and to analyse the investigational drugs.

Material and methods An observational descriptive retrospective study was carried out in a tertiary academic hospital. All active CT in the neuropsychology service from 1 January 2014 to 31 March 2019 were reviewed. Collected data were total number of CT; total number of included patients; demographic data; total number of CT classified by CT status (active/closed); clinical trial phase; therapeutic targets (reduction of amyloid plaques (AP)/precursor amyloid peptide (PAP) attack/inhibition of GLYT1 transporter/selective antagonism of 5-HT6 receptor/partial selective agonism of $\alpha_{7}$ nicotinic receptor); administration route (oral/intravenous/subcutaneous); clinical trials with results; and type of result (positive/negative).

Results Twelve CT were analysed involving a total of 59 patients (mean 5 patients per clinical trial (rank 0-8)), 34 $(57.6 \%)$ women with a mean age of 77.4 years $(95 \%$ CI 71.5-84.7). Six (50.0\%) CT were active; 3 (25.0\%) CT were phase II trials and 9 (75.0\%) were phase III trials. Therapeutic targets were reduction in AP 5 (41.7\%), attack of PAP 3 (25.0\%), inhibition of GLYT1 transporter 1 (8.3\%), selective antagonism of 5-HT6 receptor $2(16.7 \%)$, partial selective agonism of $\alpha_{7}$ nicotinic receptor $1(8.3 \%)$; route of administration oral $7(58.3 \%)$, intravenous $1(8.3 \%)$ or subcutaneous $4(33.3 \%)$; and $3(25.0 \%)$ CT had results, all of which were negative (3 (100\%)).

Conclusion and relevance

- The highest number of active CT were phase III trials.

- Only $25 \%$ of CT had results and all were negative.

- Almost $60 \%$ of CT studied oral administration, which was patients' preference.

- There were a total of five therapeutic targets but more than $40 \%$ of the CT evaluated the reduction in APs.

- Based on these results, we should rethink the research on Alzheimer's disease before continuing to develop clinical trials with the same therapeutic target.

\section{REFERENCES AND/OR ACKNOWLEDGEMENTS}

No conflict of interest.

\section{CPS-141 EFFECTIVENESS OF IMMUNOTHERAPY IN SEVERE UNCONTROLLED ASTHMA}

M Bitlloch Obiols*, L Perez Cordon, J Delgado Rodriguez, A Sanchez Ulayar, V Aguilera Jimenez, M Pujal Herranz, T Gurrera Roig, D Lopez Faixo, C Agusti Maragall, L Campins Bernadas. Hospital De Mataró, Pharmacy, Mataró, Spain

10.1136/ejhpharm-2020-eahpconf.242

Background and importance It is estimated that asthma affects $4.9 \%$ of adults in Spain and 3.9\% are classified as severe uncontrolled asthma (SUA). Omalizumab, mepolizumab, reslizumab and benralizumab are monoclonal antibodies indicated in the treatment of SUA in adults.

Aim and objectives To analyse the effectiveness and improvement in quality of life in patients with SUA treated with monoclonal antibodies in a second level hospital.

Material and methods A retrospective observational study was conducted of all patients with SUA who received monoclonal antibody therapy from August 2011 to September 2019. Age, gender and clinical data (treatment duration, ingestion of oral corticosteroids (OCS), asthma control test (ACT), presence of exacerbations requiring OCS and hospitalisations related to asthma) were recorded before starting immunotherapy and at the last follow-up visit. Effectiveness was evaluated as a reduction in OCS, exacerbations and/or hospitalisations. ACT was used to evaluate improvement in quality of life, with a score of at least 20 considered good control of asthma.

Results Forty-eight patients were included, 70.8\% ( $\mathrm{n}=34)$ were women, mean age was 56 years (23-79), and 75\% $(n=36)$ were treated with omalizumab, $18.7 \%(\mathrm{n}=9)$ with mepolizumab, 4.2\% $(n=2)$ with reslizumab and $2.1 \%(n=1)$ with benralizumab. Mean duration of treatment was $31,9,8$ and 1 month, respectively. Effectiveness was not evaluated in three patients due to lack of information. Treatment was discontinued in 7 patients for inefficacy, 3 for tolerance, 1 for adherence and 1 for hospital referral. Three patients were switched from omalizumab to mepolizumab during the study. Before starting immunotherapy, 24.3\% $(n=10)$ of patients had ACT $>20$, and in the previous year $53.5 \% \quad(n=23)$ took OCS, $83.7 \%(\mathrm{n}=36)$ had exacerbations requiring OCS and $37.2 \%$ $(n=16)$ required at least one hospitalisation due to an exacerbation. After treatment, the last follow-up results were $65.1 \%$ $(\mathrm{n}=28), 23.3 \% \quad(\mathrm{n}=10), 44.7 \% \quad(\mathrm{n}=17) \quad$ and $5.3 \% \quad(\mathrm{n}=2)$, respectively.

Conclusion and relevance Immunotherapy was effective in most cases, reducing exacerbations and hospitalisations in SUA. It also allowed discontinuation of OCS therapy. The improvement in quality of life was proved with the increase in ACT score, despite its subjectivity.

\section{REFERENCES AND/OR ACKNOWLEDGEMENTS}

No conflict of interest.

\section{CPS-142 EFFECTIVENESS AND SAFETY OF ANTI-IL-5 BIOLOGIC AGENTS IN SEVERE EOSINOPHILIC ASTHMA}

M Calvo Arbeloa*, G Pinilla Lebrero, E Lacalle Fabo, J Polo García, L Ulacia Epelde, J Illodo Becerra, D Fresán Restituto, P Aldave Cobos, J Elizondo Armendáriz, M Sarobe Carricas. Complejo Hospitalario De Navarra, Pharmacy Department, Pamplona, Spain

\subsection{6/ejhpharm-2020-eahpconf.243}

Background and importance Severe uncontrolled asthma is characterised by poor control despite treatment with inhaled glucocorticoids (IGC) and beta $_{2}$ adrenergic agonists (LABA) at high doses, and/or oral glucocorticoids (OGC). This type of asthma comprises a heterogeneous group of phenotypes treated with targeted therapy. Anti-IL-5 monoclonal antibodies (mepolizumab, reslizumab and benralizumab) are indicated in severe eosinophilic asthma (SEA).

Aim and objectives To assess the effectiveness and safety of anti-IL-5 biologic agents in a tertiary level hospital.

Material and methods This retrospective observational study included patients with SEA receiving treatment with anti-IL-5 agents from June 2017 to August 2019. Electronic clinical records were used to obtain sociodemographic variables (age, sex, concomitant medicines and previous biologicals), effectiveness (reduction in eosinophil blood levels, change in levels of exhaled nitric oxide (FeNO), forced expiratory volume in $1 \mathrm{~s}$ $\left(\mathrm{FEV}_{1}\right)$ and score in the asthma control test (ACT)) and safety (reported adverse effects).

Results Thirty-four patients were included, 67.6\% (23) women, and mean age was 56.2 (41-69) years. Twenty patients received mepolizumab with an average duration of 40 weeks, 4 reslizumab for 27.7 weeks and 9 benralizumab for 